\title{
CONEXÕES POLÍTICAS E DESEMPENHO ECONÔMICO: UM ESTUDO COM AS EMPRESAS DO SEGMENTO DE ENERGIA ELÉTRICA LISTADAS NA B3
}

\section{POLITICAL CONNECTIONS AND ECONOMIC PERFORMANCE: A STUDY OF THE ELECTRICITY COMPANIES LISTED ON BRAZILIAN STOCK EXCHANGE (B3)}

\begin{abstract}
RESUMO
Este trabalho teve o objetivo de identificar e descrever a relação entre a conectividade política e o desempenho econômico das empresas do segmento de energia elétrica listadas na B3. Foram analisadas 26 empresas cujos dados foram coletados no conjunto de demonstrações contábeis, nos formulários de referência arquivados na CVM e nas cotações históricas obtidas no sítio eletrônico da B3, entre os anos de 2013 e 2018, utilizando a técnica de regressão quantílica para relacionar o desempenho econômico com a conectividade política, o tamanho, liquidez corrente, endividamento e governança corporativa. Como resultados foi possível observar que a conexão política por presença na propriedade se mostrou significante e positivamente relacionada com o desempenho econômico, ao passo que não foram encontradas evidências suficientes para comprovar que há relação entre o desempenho econômico e a conexão política, por meio da presença no conselho de administração. A presente pesquisa contribui para que investidores minoritários tenham uma melhor percepção sobre a relação contratual de agência com o governo ao evidenciar que há uma relação positiva entre o desempenho econômico mensurado pelo Q de Tobin e o Market-to-book e a conectividade política exercida por meio da presença do governo como acionista, controlador ou com influência significativa.
\end{abstract}

Palavras-chave: Conexões políticas; Conselhos de administração; Desempenho econômico.

\section{ABSTRACT}

This research aimed to identify and describe the relationship between political connectivity and the economic performance of electricity companies listed on Brazilian stock exchange (B3). We analyzed 26 companies whose data were collected in the set of financial statements and in the CVM's Form-20F and in the historical prices on the B3 website, for the period between 2013 and 2018, using the quantile regression technique to analyze the relation between economic performance and political connectivity, current liquidity ratio, firm size, leverage and corporate governance firm level. As a result, it was possible to observe that the political connection by ownership presence shown to be significantly and positively related to economic performance, whereas insufficient evidence was found to prove that there is a relationship between economic performance and political connection measured through presence on the board of directors. This research contributes to minority investors to have a better perception of agency contractual relationships with the government by showing that there is a positive relationship between the economic performance measured by Tobin's Q and the Market-to-book with political connectivity exercised through the presence of the government as a shareholder, controller or with significant influence.

Keywords: Political connections; Boards of directors; Economic performance.

\section{Robson dos Santos Costa}

Mestre em Inovação e Desenvolvimento pelo Centro Universitário dos Guararapes (UNIFG). Especialista em Contabilidade e Controladoria Empresarial pela Universidade Federal de Pernambuco (UFPE). Graduado em Ciências Contábeis pela Faculdade de Ciências Contábeis do Recife (FACCOR). Docente dos cursos de Ciências Contábeis e Administração na Faculdade de Ciências Humanas de Olinda - FACHO. E-mail: rsc_robson@hotmail.com

\section{Juliana Gonçalves de Araújo Doutoranda em Ciências Contábeis na Universidade Federal de Pernambuco (UFPE). Doutora em Administração pela Universidade Federal de Pernambuco (UFPE). Graduada em Ciências Contábeis Universidade Federal de Pernambuco (UFPE). Docente do curso de Administração na Universidade de Pernambuco (UPE). E-mail: juhliana.araujo@gmail.com}

\section{Rodrigo Vicente dos Prazeres} Doutor em Ciências Contábeis pela Universidade Federal de Pernambuco (UFPE). Graduado em Ciências Contábeis pela Universidade Católica de Pernambuco (UNICAP). Docente da Faculdade de Economia, Administração e Contabilidade (FEAC) da Universidade Federal de Alagoas (UFAL). E-mail: rodrigo.prazeres@feac.ufal.br

\section{João Gabriel Nascimento de Araújo \\ Mestre em Ciências Contábeis pela Universidade Federal de Pernambuco (UFPE). Graduado em Ciências Contábeis Universidade Federal de Pernambuco (UFPE). Docente na Faculdade Asces-Unita. E-mail: jgabriel90@hotmail.com}




\section{INTRODUÇÃO}

À medida que as relações empresariais se tornaram mais complexas, houve uma separação entre a propriedade e o controle nas organizações (Berle \& Means, 1932). Neste cenário, uma ou mais pessoas (principal) contratam alguém (agente) para que este tome decisões sobre determinada atividade empresarial. Estes detentores do capital (investidores) transferem a terceiros a missão de maximização dos recursos de seus empreendimentos (Jensen \& Meckling, 1976).

Segundo Jensen \& Meckling (1976) uma empresa é formada por uma rede de relações contratuais (explícitas ou não) das quais se instituem funções e criam direitos e deveres entre as partes relacionadas. Destas relações verificam-se que há interesses distintos na otimização de resultados dos agentes econômicos em virtude de suas propensões ao risco. Ao principal, o interesse em maximizar o retorno pelos seus investimentos, enquanto para o agente a otimização de seus ganhos como executivo (remuneração fixa, variável e bonificações). Dessas situações, por vezes contraditórias, surgem conflitos de agência decorrentes de problemas de assimetria informacional (Jensen \& Meckling, 1976).

Na maioria dos países, incluindo o Brasil, a figura do acionista majoritário (detentor do controle, e consequentemente, do poder de indicação dos gestores) é a mais presente, em função de distúrbios no mercado, o que leva o controlador a concentrar propriedade como forma de proteção e de manutenção de poder (La Porta, Lopez-de-Silanes, Shleifer \& Vishny, 1997). Já o modelo de propriedade acionária pulverizado, descrito por Berle \& Means (1932), aplica-se a nações como Estados Unidos e Grã-Bretanha, por exemplo, sobretudo, por conta do estágio de desenvolvimento do mercado de capitais, onde há um maior enforcement e prerrogativas legais que garantem maior proteção legal às relações contratuais (La Porta et al., 2000).

La Porta et al. $(1998,2000)$ explicam que as estruturas de propriedade das empresas são afetadas por diversos elementos e dentre os quais a proteção legal derivada de mecanismos externos de governança corporativa. Há uma relação positiva entre medidas protetivas entre agente e principal com uma maior dispersão acionária e o estágio de desenvolvimento do mercado financeiro. A negativa de tal proposição resulta em um mercado financeiro com menor enforcement e maior concentração acionária, favorecendo conflitos de interesses entre os acionistas minoritários e majoritários (Thomsen \& Pedersen, 2000; Okimura, Silveira \& Rocha, 2007, Caixe \& Krauter, 2013).

Neste ambiente, as conexões políticas podem se fazer presentes em empresas de capital aberto, em especial, aquelas com estrutura de propriedade concentrada, justamente por reduzirem o risco por meio de informações privilegiadas, tendo como premissa que a proteção legal é insuficiente ou não confiável (Faccio, 2006). A concentração de direitos de controle está relacionada com a obtenção de vantagens informacionais pelos majoritários em relação aos minoritários e uma das formas de obtê-las é por meio das conexões políticas, principalmente em economias menos desenvolvidas, pois visa proteger as empresas de privações pelo governo e acesso aos subsídios destes e podem variar entre paises e entre regiões de um mesmo país (Chen, Su \& Sun, 2011), bem como no seu desempenho econômico (Silva, Xavier, Gambirage \& Camilo 2018).

As conexões políticas podem surgir por intermédio da estrutura de propriedade de maneira direta, quando o governo é um acionista, ou quando a empresa possui acionistas decisores políticos ou quando ex-políticos integram a gestão ou o conselho de administração das organizações; e de maneira indireta ou por bancos públicos que fomentam o desenvolvimento ou por fundos de pensão de empresas estatais (Bandeira de Mello \& Marcon, 2011; Brey, Camilo, Marcon \& Alberton, 2011). Outras formas, de conexões políticas são por meio de atividades de lobby (Hill, Fuller, Kelly \& Washam, 2014; Chen, Parsley \& Yang, 2015); por meio de doações de campanhas eleitorais (Camilo, Marcon \& Bandeira de Mello, 2012; Pinheiro, De Luca \& Vasconcelos, 2016); critérios geográficos (Faccio \& Parsley, 2009; Chen et al.,2011); ou, quando um dos membros do conselho de administração ocupa a posição de conselheiro em mais de uma empresa simultaneamente (board interlocking) (Barringer \& Harrison, 2000; Fich \& White, 2005; Camilo et al., 2012).

Para este estudo, a conectividade política foi estudada por meio da estrutura de propriedade em que há a participação do governo como acionista e por meio da participação de membros do conselho de administração com conexões políticas (La Porta et al., 2002; Claessens, Feijen \& Laeven, 2008, Bandeira de Mello \& Marcon, 2011, Brey et al., 2011, Camilo et al., 2012; Dinç, 2005, Macêdo, 2017). Brey, Camilo, Marcon \& Bandeira de Mello (2014) afirmam que conexões políticas favorecem a criação de mecanismos de barreira de entrada de concorrentes (nacionais ou estrangeiros) ou mesmo dificultam aberturas de novos mercados que possam prejudicar os negócios da organização. Esta pesquisa estudou a conectividade política no segmento de energia elétrica em empresas listadas na Brasil, Bolsa, Balcão (B3). A seleção do segmento econômico ocorreu em virtude das características específicas deste negócio: sua alta regulação, importância dentro da cadeia produtiva, e relevância social deste nicho e a grande quantidade de organizações que negociam ações na bolsa de valores brasileira.

Com base nos argumentos aqui expostos, esta pesquisa visa responder o seguinte questionamento: qual a relação entre a conectividade política e o desempenho econômico das empresas do segmento de energia elétrica listadas na B3?

Diante do exposto, este trabalho tem por objetivo identificar e descrever a relação entre a conectividade política e o desempenho econômico das companhias do segmento de energia elétrica da B3.

O setor elétrico detém importante papel dentro da cadeia produtiva na geração de bens e serviços para a sociedade. Entretanto, por questões legais, este segmento é impedido de conectar-se politicamente por doações a campanhas, o que favoreceu o aprofundamento das conexões políticas por meio de membros em conselhos de administração e da estrutura 
de propriedade. A partir dessa constatação, vislumbra-se a oportunidade de estudar a relação entre a conectividade política e o desempenho econômico com o intuito de avançar na compreensão do estabelecimento das relações contratuais de agência em um segmento regulado da B3.

\section{REFERENCIAL TEÓRICO}

\subsection{Conexões Políticas}

Baysinger (1984) cita três objetivos principais das conexões políticas nas corporações: (1) obter favores de cunho financeiro e de restrição à competição junto aos governos, ou seja, refere-se à tentativa de uma organização de usar os poderes do governo para perseguir seus próprios interesses, geralmente em detrimento de outras empresas ou da sociedade em geral; (2) gerenciar possíveis ameaças governamentais à legitimidade dos objetivos organizacionais e à defesa do domínio de propósitos empresariais. Assim, as conexões políticas visam influenciar políticas públicas que ameacem a legitimidade dos objetivos e propósitos organizacionais; e (3) monitorar ameaças concorrenciais que possam comprometer a manutenção de domínio, especialmente aquelas apresentadas pelo governo como possíveis regulamentações governamentais que sejam divergentes aos métodos gerenciamento escolhidos pela organização.

No Brasil, o processo de privatizações em companhias estatais na década de 1990 proporcionou um novo arranjo na estrutura de propriedade destas organizações. Apesar da privatização e desregulamentação ter sido uma tendência global, as empresas, nos tempos atuais, ainda apresentam forte dependência externa do governo para minimizar as incertezas. Esta dependência leva à formação de conexões políticas (Brey et a.l, 2011).

O desenvolvimento histórico da relação política entre empresas e governo ocorreu de forma não-linear (Shaffer, 1995). Em virtude disso, diversas nomenclaturas foram utilizadas para tratar deste fenômeno social, a citar alguns exemplos: "estratégias políticas corporativas" indicadas por Hillman \& Hitt (1999); "atividades políticas corporativas" em Baysiner (1984) e Shaffer (1995); e "estratégias não mercantis” em Bandeira de Mello \& Marcon (2011).

Esta pesquisa utiliza a expressão "conexão política” ou "conectividade política" ao abordar este fenômeno social. Faccio (2006, p. 6) entende que há conexão política quando "uma empresa está conectada a um político se (pelo menos) um dos grandes acionistas da empresa ou alto executivo é (1) um membro do parlamento (2), um ministro ou o chefe de Estado, ou (3) está intimamente relacionado com um oficial do alto escalão”.

Há situações de conexão política por intermédio da estrutura de propriedade de maneira direta, quando o governo é um acionista, ou quando a empresa possui acionistas decisores políticos e/ou quando políticos aposentados fazem parte das empresas como gestores ou membros dos conselhos; e de maneira indireta ou por bancos públicos que fomentam o desenvolvimento ou por fundos de pensão de empresas estatais (Bandeira de Mello \& Marcon, 2011, Brey et al., 2011).

Para Chen et al. (2011), na busca por recursos através de conexões políticas, empresas tendem a apresentar estrutura de propriedade concentrada para garantir o processo de decisão por trazer: (1) maior homogeneidade do controle de interesses, reduzindo custos em ações coletivas para criar e manter conexões políticas; (2) os benefícios das conexões políticas gerados terão menor diluição do que seriam numa organização com concentração acionária mais dispersa; (3) redução nos custos de transmissão de informação entre acionistas.

As conexões políticas oferecem vantagens competitivas para empresa no incremento do seu valor. Por meio delas, é possível acesso preferencial a recursos e capitais, a benefícios fiscais, ao favorecimento em contratos com o governo, e à flexibilização de regulações dentre outras (Faccio, 2006). Hillman \& Hitt (1999) citam três estratégias das organizações quanto ao dilema das incertezas e dependência externas: (1) obter informação com decisores políticos; (2) contribuir com campanhas eleitorais e estreitar vínculos; (3) ter membros internos na empresa que votem de acordo com as metas institucionais. Cada estratégia poderá ser utilizada isoladamente ou combinadas dependendo do interesse proposto.

A terceira vertente foi uma das exploradas por esta pesquisa com destaque aos membros dos conselhos de administração. Esta ótica de participação em conselhos, de acordo com Cooper, Gulen \& Ovtchinnikov (2010), é uma das formas mais presentes nos estudos internacionais sobre o tema, com formas mais indiretas como, por exemplo, contribuições a políticos, que será explorado adiante.

Por questões legais no Brasil, entidades de utilidade pública, nos quais o setor de energia elétrica está inserido, são proibidas de realizar doações de maneira direta ou indireta partidos políticos ou candidatos, conforme artigo 24 da Lei $\mathrm{n}^{\circ}$ 9.504, de 30 de setembro de 1997. O que justifica o direcionamento para os conselhos de administração e estrutura de propriedade como elos de conexões políticas.

\subsection{Conexões Políticas por Estrutura de Propriedade}

Esta forma de conectividade política refere-se à participação do governo como acionista controlador ou com participação significativa em uma empresa. De acordo com Macêdo, Silva \& Machado (2015), as conexões políticas por estrutura de propriedade são tipificadas de maneira explícita, quando o governo e suas organizações são acionistas da empresa; e implícita quando há participação em empresas por meio de estrutura piramidal, ou seja, o governo é ,sócio de uma empresa e essa é proprietária de outra.

Sobre os conflitos de agência em empresas com conexões políticas através de acionista majoritário, Wu, Wu \& Rui 
(2010) afirmam que é mais agravante do que em instituições sem conectividade política. Os autores justificam que estes acionistas controladores podem tomar decisões divergentes à maximização do valor da empresa em favor dos objetivos macro do governo (Wu et al., 2010). Arrow (1985) explica que, para o agente, há um interesse em ocultar informações com a finalidade de impedir que o principal visualize o seu desempenho econômico de forma mais coerente. Com essa assimetria informacional, as conexões políticas podem se fazer presentes em empresas de capital aberto, em especial, aquelas com estrutura de propriedade concentrada, justamente por reduzirem o risco por meio de informações privilegiadas (Faccio, 2006); e por possibilitaram um maior controle decisório que favorece o aumento dos benefícios privados de controle dos acionistas majoritários (Chen et al, 2011).

\subsection{Conexões Políticas por Conselho de Administração}

Não há, atualmente, um consenso sobre a definição de conectividade em conselhos de administração (Menozzi, Gutiérrez Urtiaga \& Vannoni, 2011). Uma das mais usuais classificações é a de que um membro do conselho é considerado como politicamente conectado quando é integrante do parlamento, chefe de Estado, possui ligação direta com partidos políticos ou tem amigos ou parentes próximos nestas condições (Faccio, 2006). Para Boubakri, Cosset \& Saffar (2008, p. 657): "uma empresa é politicamente ligada se pelo menos um membro do seu Conselho de Administração, inclusive o CEO e Conselho Fiscal é ou foi um político, isto é, um membro do parlamento, um ministro ou qualquer outro alto burocrata”. Já Menozzi et al. (2011) têm uma definição mais flexível. Para eles, há conexões políticas nas empresas, quando um ou mais de seus colaboradores estão relacionados com alguma atividade, presente ou passada, por nomeação política, filiação partidária ou candidatura para cargo público.

Faccio (2006) argumenta que conexões políticas por meio de conselhos de administração, executivos e acionistas são mais sólidas do que doações esporádicas ou mesmo outras transferências de recursos. Conselhos politicamente conectados podem influenciar nos resultados das companhias eleitorais (Salamon \& Siegfried, 1977). Segundo Ecco (2010, p. 20) "os conselheiros políticos podem agir no sentido de sensibilizar o governo para atuar nos interesses das empresas e preveni-las sobres ações hostis do governo”. E como uma estratégia para a troca de informações privilegiadas e a possibilidade de compra de influências reduzindo incertezas (Hillman, 2005; Hillman, Zardkoohi \& Bierman, 1999).

Em seu estudo, Chen et al. (2011) definiram como um membro de conselho conectado politicamente aquele satisfizer pelo menos um dos seguintes critérios: (1) ex-funcionário do governo; (2) atual ou antigo membro do Congresso do Povo; (3) atual ou antigo membro da Conferência Consultiva Política do Povo. Os itens dois e três referem-se a influentes organizações quase governamentais no sistema político chinês.

Por conseguinte, na próxima seção é apresentado o corpo de evidências empíricas sobre a presente temática. O propósito desta seção é o de justificar o embasamento teórico das relações entre o desempenho econômico e a conectividade política.

\subsection{Estudos Anteriores}

Nesta seção são apresentados estudos nacionais e internacionais que investigaram a relação entre a conectividade política e o desempenho econômico, com foco nos principais objetivos e resultados observados.

Hillman (2005) percebeu uma relação positiva com a performance de mercado em empresas cujos conselhos de administração são conectados, principalmente em setores regulados (a autora analisou dois grupos de empresas com grande e outro com pouca regulação), constando que em ambiente com maior regulação há maior concentração de conselheiros com experiência política, quando compara-se ao grupo com menor regulação.

Ecco (2010) avaliou o desempenho econômico e conectividade política através dos conselhos de administração de 127 empresas de cinco setores da bolsa de valores brasileira (Petróleo, Gás e Biocombustíveis; Materiais Básicos; Construção e Transporte; Telecomunicações; e Utilidade Pública), no período de 1999 a 2008. Em seu estudo, constatou que o segmento de utilidade pública possui o maior número de empresas conectadas e, consequentemente, o maior número de conselheiros com experiência política. Observou ainda que não foram apresentadas relações estatísticas significativas com o desempenho econômico e a adoção dessas estratégias políticas corporativas.

Menozzi et al. (2011) buscaram compreender se a composição dos conselhos de administração com conexões políticas de empresas estatais italianas de serviços públicos de gás, água, eletricidade e transporte público, no período de 1994 a 2004, impactam na demanda por emprego e desempenho econômico destas organizações. Concluíram quetais instituições com estes representantes geram mais empregos, contudo apresentam desempenho econômico negativo.

Chen et al. (2011) estudaram 276 empresas chinesas privadas listadas nas duas bolsas de valores do país (Xangai e Shenzhen) entre 1993 e 2008. Na seleção destas organizações, levaram em conta instituições não estatais (para evitar amostras com conexões políticas impostas pelo governo). Os autores observaram que empresas ligadas a regiões com mais incentivos governamentais são mais propícias a construir conexões políticas e como essas conexões afetam as estruturas de controle destas empresas. Também detectaram que empresas com maior conectividade política (com representantes, ou no conselho de administração, ou como representante da empresa) apresentam controle acionário mais concentrado do que as não conectadas.

Camilo et al. (2012) analisaram empresas não financeiras listadas na bolsa brasileira de 1998 a 2009, de acordo com mandatos presidenciais utilizando as três proxies mais usuais de conexão política (doação para campanhas; membro 
de conselho ou diretoria com experiência política; e conselheiro que participa de múltiplas companhias). No estudo, constataram uma relação positiva entre conectividade política e o desempenho econômico, pois a conectividade com o ambiente político se mostrou positivamente relacionada ao desempenho econômico mensurado pelo $Q$ de Tobin e Market-to-book.

Jackowicz, Kozlowski \& Mielcarz (2014) investigaram o impacto das conexões políticas através de membros do conselho de administração e diretores com o desempenho econômico em 316 empresas não financeiras polonesas listadas na bolsa de valores da Varsóvia, nos anos de 2001 a 2011, tendo como proxies os membros do conselho de administração e diretores. Entre seus achados, constataram que quase um terço da amostra apresentaram conectividade e índices de rentabilidade inferiores quando comparada a outra subamostra.

Já Macêdo (2017), ao estudar sobre o impacto da mutualidade entre bancos e governo, por meio das conexões políticas, no desempenho econômico dos bancos em operação no Brasil com as mesmas proxies de conexão usadas por Camilo et al. (2012). Constatou que, $71 \%$ dos bancos apresentaram conexão política através de doação dando maior estabilidade ao desempenho econômico medido pelo retorno sobre o patrimônio líquido; e que a variável conexão e sua influência persistem em até cinco períodos semestrais após eleição.

Sintetizando os resultados apresentados nessa seção, é possível observar que no Brasil foi constatada uma alta conectividade política das organizações que atuam em setores regulados (ECCO, 2010), a exemplo do segmento de energia elétrica; e, uma relação positiva entre o desempenho econômico e a conectividade política (CAMILO et. al., 2012; MACÊDO, 2017). No mesmo sentido, o corpo de evidências empíricas internacionais confirma que há maior conectividade política em setores com maior regulação (HILLMAN, 2005) e em empresas que fazem uso de incentivos estatais (CHEN et al., 2011), mas diverge dos resultados apresentados das pesquisas nacionais ao evidenciar relações negativas entre o desempenho econômico (JACKOWICZ et. al., 2014) e a rentabilidade (MENOZZI et al., 2011) das organizações que possuem algum tipo de conectividade política.

\section{METODOLOGIA}

\subsection{População e Amostra}

Para a delimitação amostral, foram consideradas as seguintes premissas: i) a empresa estar inserida no segmento de energia elétrica; ii) possuir histórico completo de demonstrações contábeis arquivadas no sítio eletrônico da Comissão de Valores Mobiliários (CVM) entre os anos de 2013 e 2018; iii) apresentar ações em circulação que permitam obter o seu valor de mercado no período do estudo; iv) não apresentar patrimônio líquido negativo v) possuir o histórico de formulários de referência arquivados no site da CVM com status "Ativo" para o período do estudo.

Assim, das 59 empresas constantes do segmento de energia elétrica foi possível a coleta de dados referentes a 26 empresas, o que representa $44,07 \%$ da população conforme a tabela 1 :

Tabela 1- Amostra da pesquisa

\begin{tabular}{c|c|c|c}
\hline Ordem & Razão Social & Nome de Pregão & Nível de Governança \\
\hline 1 & AES Tiete Energia SA & AES TIETE E & Nível 2 \\
2 & Ampla Energia e Serviços S.A & AMPLA ENERG & Segmento Tradicional \\
3 & Centrais Ele Bras S.A - Eletrobras & ELETROBRAS & Nivel 1 \\
4 & Centrais Ele de Santa Catarina S.A & CELESC & Nível 2 \\
5 & CESP- Cia Energética de São Paulo & CESP & Nível 1 \\
6 & Cia Energética de Brasília & CEB & Segmento Tradicional \\
7 & Cia Energética de Minas Gerais - CEMIG 1 \\
8 & Cia Energética do Ceará - COELCE & CEMIG & Segmento Tradicional \\
9 & Cia Energética do Maranhão - CEMAR & CEMAR & Mercado de Balcão \\
10 & Cia Energética do Rio Gde Norte - COSERN & COSERN & Segmento Tradicional \\
11 & Cia Estadual Ger. Trans. Ener. Elet - CEEE-GT & CEEE-GT & Nível 1 \\
12 & Cia Paranaense de Energia - COPEL & COPEL & Nível 1 \\
13 & CPFL Energia S.A & CPFL ENERGIA & Novo Mercado \\
14 & CPFL Energias Renováveis S.A & CPFL RENOVAV & Novo Mercado
\end{tabular}




\begin{tabular}{c|c|c|c}
\hline Ordem & Razão Social & Nome de Pregão & Nível de Governança \\
\hline 15 & CTEEP - Cia Transmissão Energia Elétrica Paulista & TRAN PAULIST & Nível 1 \\
16 & EDP - Energias do Brasil S.A & ENERGIAS BR & Novo Mercado \\
17 & Elektro Redes S.A & ELEKTRO & Segmento Tradicional \\
18 & Eletrobrás Participações S.A - ELETROPAR & ELETROPAR & Segmento Tradicional \\
19 & EMAE - Empresa Metrop. Aguas Energia S.A & EMAE & Segmento Tradicional \\
20 & Energisa Mato Grosso - Distribuidora de Energia S/A & ENERGIA MT & Segmento Tradicional \\
21 & Energisa S.A & ENERGISA & Nível 2 \\
22 & Eneva S.A & ENEVA & Novo Mercado \\
23 & Equatorial Energia S.A & EQUATORIAL & Novo Mercado \\
24 & Light S.A & LIGHT S/A & Novo Mercado \\
25 & Neoenergia S.A & NEOENERGIA & Novo Mercado \\
26 & Rio Paranapanema Energia S.A & GER PARANAP & Segmento Tradicional \\
\hline
\end{tabular}

Fonte: B3 (2021).

\subsection{Mensuração das Variáveis do Estudo}

\subsubsection{Variáveis Dependentes}

De acordo com a literatura, nessa pesquisa são utilizadas duas das variáveis mais comuns para mensurar o desempenho econômico, quais sejam: i) $Q$ de Tobin; e ii) Market-to-Book. Na tabela 2, são apresentadas as variáveis dependentes e suas respectivas mensurações.

Tabela 2 -Variáveis dependentes

\begin{tabular}{c|c|c|c}
\hline Variável & Mensuração da Variável & Referências & Fonte dos dados \\
\hline $\begin{array}{c}\text { Q de Tobin } \\
\text { (QTobin) }\end{array}$ & $\begin{array}{c}\text { Razão entre o valor de mercado somado ao } \\
\text { passivo exigível e os ativos totais }\end{array}$ & $\begin{array}{c}\text { Chung \& Pruitt (1994), } \\
\text { Camilo et al. (2012). }\end{array}$ & $\begin{array}{c}\text { Demonstrações contábeis e } \\
\text { dados de mercado }\end{array}$ \\
$\begin{array}{c}\text { Market-to-Book } \\
(M T B)\end{array}$ & $\begin{array}{c}\text { Razão entre o valor de mercado e patrimônio } \\
\text { líquido }\end{array}$ & $\begin{array}{c}\text { Camilo et al. (2012). } \\
\text { Carroch (2004), }\end{array}$ & $\begin{array}{c}\text { Demonstrações contábeis e } \\
\text { dados de mercado }\end{array}$ \\
\hline
\end{tabular}

Fonte: elaboração própria (2021).

Todos os dados contábeis necessários para o cálculo das variáveis supramencionadas foram coletados diretamente do conjunto de demonstrações contábeis arquivadas no sítio eletrônico da Comissão de Valores Mobiliários (CMV). Já os dados dos preços das ações para o cálculo do valor de mercado foram obtidos no sítio eletrônico da B3.

\subsubsection{Variáveis Independentes}

Para as variáveis independentes, as proxies representativas da conectividade política foram coletadas a partir da análise documental dos formulários de referência, de cada companhia, obtidos no sítio eletrônico da CMV. A seguir a tabela 3 sintetiza as variáveis independentes:

Tabela 3 -Descrição das variáveis independentes

\begin{tabular}{c|c|c|c}
\hline Variável & $\begin{array}{c}\text { Sinal } \\
\text { esperado }\end{array}$ & Descrição da variável & Referência teórica \\
\hline $\begin{array}{c}\text { Conexão Geral } \\
\text { Dummy } \\
\text { (ConexG) }\end{array}$ & $(+)$ & $\begin{array}{c}\text { Indica se houve conexão política identificada. } \\
\text { Variável Dummy. Assume (1) conexão política por } \\
\text { estrutura de propriedade ou presença em conselhos } \\
\text { de administração (0) na ausência da conexão política. }\end{array}$ & $\begin{array}{c}\text { Dinç (2005), Claessens et al. (2008), } \\
\text { Brey et al. (2011), Camilo et al. } \\
\text { (2012), Brey et al. (2014) }\end{array}$
\end{tabular}




\begin{tabular}{|c|c|c|c|}
\hline Variável & $\begin{array}{c}\text { Sinal } \\
\text { esperado }\end{array}$ & Descrição da variável & Referência teórica \\
\hline $\begin{array}{l}\text { Conexão } \\
\text { Propriedade } \\
\text { Dummy } \\
\text { Nível } 1 \\
\text { (ConexPro) }\end{array}$ & $(+)$ & $\begin{array}{l}\text { Indica o nível de conexão política identificada. } \\
\text { Assume (0) se não conectado e (1) se conectado via } \\
\text { estrutura de propriedade. }\end{array}$ & $\begin{array}{c}\text { Claessens et al. (2008), Brey et al } \\
\text { (2011), Bandeira de Mello \& Marcon } \\
\text { (2011), Chen et al (2011), Camilo et } \\
\text { al. (2012), Brey et al (2014), Macêdo } \\
\text { et al. (2015). }\end{array}$ \\
\hline $\begin{array}{l}\text { Conexão } \\
\text { Presença } \\
\text { Dummy } \\
\text { Nível 2 } \\
\text { (ConexPre) }\end{array}$ & $(+)$ & $\begin{array}{l}\text { Indica o nível de conexão política identificada. } \\
\text { Assume (0) se não conectado e (1) se conectado via } \\
\text { estrutura de governança: presença de um ex-político } \\
\text { no conselho de administração. }\end{array}$ & $\begin{array}{l}\text { Dinç (2005), Claessens et al. (2008), } \\
\text { Brey et al (2011), Menozzi et al. } \\
\text { (2011), Camilo et al. (2012); Brey et al } \\
\text { (2014), Macêdo (2017). }\end{array}$ \\
\hline
\end{tabular}

Fonte: elaboração própria (2021)

Com base nos resultados do estudo de Camilo et al. (2012), que constataram que há relação positiva entre a conectividade política e o desempenho econômico, é lançada a primeira hipótese de pesquisa:

$\mathrm{H}_{1}$ : Há uma relação positiva entre a conectividade política com o desempenho econômico das empresas do segmento de energia elétrica listadas na B3.

A conectividade política destacada na $\mathrm{H}_{1}$ é analisada em dois desdobramentos. A primeira tendo o governo como acionista (Claessens et al., 2008, Brey et al., 2011, Bandeira de Mello \& Marcon 2011, Chen et al., 2011, Camilo et al., 2012, Brey et al., 2014) sendo a estrutura de propriedade definida como explícita conforme Macêdo et al. (2015), em que é possivel apresentar o seguinte desdobramento da hipótese de estudo:

$\mathrm{H}_{1 \mathrm{a}}$ : Há uma relação positiva entre conectividade política por meio do acionista governo com o desempenho econômico das empresas do segmento de energia elétrica listadas na B3.

Esta forma de conexão política teve como proxy o acionista governo, quando este possuir influência significativa ou controle da empresa analisada, ou seja, ter pelo menos $20 \%$ do capital votante conforme a Lei $6.404 / 76$. Para obtenção destes dados, foi realizada uma análise documental dos formulários de referência de cada empresa.

A segunda forma de conectividade política foi atrelada aos conselheiros administrativos (Claessens et al., 2008, Brey et al., 2011, Bandeira de Mello \& Marcon 2011, Chen et al., 2011, Camilo et al., 2012, Brey et al., 2014). Para isto, foi adotada a seguinte hipótese com base em Menozzi et al. (2011), Chen et al. (2011) e Macêdo (2017):

$\mathrm{H}_{1 b}$ : Há uma relação positiva entre a conectividade politica por meio de membros em conselhos de administração com o desempenho econômico das empresas do segmento de energia elétrica listadas na B3.

Esta hipótese adotada tem como base a definição de conexão política por meio de conselheiros administrativos conforme Menozzi et al. (2011), Chen et al. (2011) e Macêdo (2017), ou seja, se pelo menos um membro do conselho administrativo, no período deste estudo, possuir histórico político-partidário ou for ex-funcionário do governo. A verificação destes dados se deu a partir da investigação do currículo dos conselheiros fornecido por meio dos formulários de referência. Essa etapa da pesquisa foi realizada durante a análise documental realizada para a definição das variáveis independentes apresentadas na tabela 3.

\subsubsection{Variáveis de Controle}

Para as variáveis de controle, foram selecionadas as variáveis que de acordo com a literatura possuem reconhecida relação com o desempenho econômico. Na uabela 4 são descritas as variáveis de controle.

Tabela 4 - Descrição das variáveis de controle

\begin{tabular}{c|c|c|c}
\hline Tipo & $\begin{array}{c}\text { Sinal } \\
\text { esperado }\end{array}$ & Descrição da variável & Referência teórica \\
\hline $\begin{array}{c}\text { Tamanho da } \\
\text { Empresa } \\
\text { (TAM) }\end{array}$ & $(+)$ & $\begin{array}{c}\text { Indica controle para efeito da diferença de escala referente ao } \\
\text { tamanho da empresa, no segmento de energia, definida pelo } \\
\text { logaritmo do valor de mercado. }\end{array}$ & Correia (2018)
\end{tabular}




\begin{tabular}{c|c|c|c}
\hline Tipo & $\begin{array}{c}\text { Sinal } \\
\text { esperado }\end{array}$ & Descrição da variável & Referência teórica \\
\hline $\begin{array}{c}\text { Alavancagem } \\
\text { (ALAV) }\end{array}$ & $(-)$ & $\begin{array}{c}\text { Valores elevados de endividamento indicam maiores riscos } \\
\text { de insolvência, o que propicia um maior custo de dívida que, } \\
\text { consequentemente, compromete o desempenho econômico. } \\
\text { É mensurada pela razão entre passivo exigivel e ativo total. }\end{array}$ & $\begin{array}{c}\text { Okimura et al (2007), } \\
\text { Boubakri et al. (2008) }\end{array}$ \\
$\begin{array}{c}\text { Liquidez } \\
\text { Corrente } \\
\text { (LC) }\end{array}$ & $(+)$ & $\begin{array}{c}\text { Indica a capacidade das empresas em honrar compromissos. } \\
\text { É mensurada pela razão entre ativo e passivo circulante. }\end{array}$ & Koprowsing (2019) \\
$\begin{array}{c}\text { Governança } \\
\text { Corporativa } \\
\text { Dummy } \\
\text { (GC) }\end{array}$ & $(+)$ & $\begin{array}{c}\text { Atribui-se (1) caso a empresa esteja no nível de governança } \\
\text { N1, N2 ou NM; e (0) em caso contrário. }\end{array}$ & Correia (2018) \\
\hline
\end{tabular}

Fonte: Elaboração própria (2020).

\subsubsection{Modelagem}

Para atender ao objetivo de identificar e descrever a relação entre a conectividade política e o desempenho econômico nas empresas do setor de energia elétrica listadas na B3, foi utilizada a seguinte modelagem econométrica:

$$
\text { Desempenho }_{i, t}=\beta_{0}+\beta_{1} \text { Conexão }_{i, t}+\beta_{2} \text { TAM }_{i, t}+\beta_{3} A L A V_{i, t}+\beta_{4} L C_{i, t}+\beta_{5} G C_{i, t}+\varepsilon_{i, t}
$$

Onde:

Desempenho $_{\mathrm{i}, \mathrm{t}}$ é a variável dependente, assumindo o valor do $Q$ de Tobin ou do índice Market-to-book da empresa i no tempo t;

Conexão ${ }_{i, t}$ assume os valores das variáveis conexão geral, conexão propriedade e conexão presença da empresa i no tempo t.

Desse modo, são estimadas seis regressões para analisar as relações entre a conectividade política e o desempenho econômico. No processo de exame do conjunto de dados, foi possível verificar problemas de grande heterogeneidade que implicou em distribuições de probabilidade não normais e de variância não constante dos resíduos, caracterizando problemas de heterocedasticidade.

Com a finalidade de operacionalizar os modelos econométricos de forma confiável, foi utilizada a técnica de regressão quantílica de Koenker \& Basset (1978), que consiste em um método semiparamétrico que estima os coeficientes dos regressores a partir da mediana dos quantis, podendo ser escrito como, em que é o conjunto de coeficientes das variáveis a ser estimado, em que a estimação minimiza tornando a estimação robusta a outliers, resíduos heterocedásticos e distribuições não gaussianas.

Ademais, não foram verificados problemas de multicolinearidade entre as variáveis. Este diagnóstico foi realizado por meio do teste VIF (Variance Inflation Factor) e não foram verificados resultados superiores a dez. Por fim, todos os dados foram coletados manualmente durante os meses de junho e julho de 2019 para um horizonte temporal de pesquisa que compreende o período entre 2013 e 2018 (dados anuais), totalizando 156 observações e as relações do modelo de regressão foram analisadas para níveis de significância de $1 \%\left({ }^{* \star *}\right), 5 \%\left({ }^{* *}\right)$ e $10 \%\left({ }^{*}\right)$.

\section{ANÁLISE E DISCUSSÃO DOS RESULTADOS}

\subsection{Identificação da Conectividade Política}

Esta seção tem como finalidade identificar e descrever se há conectividade política seja pela presença do governo como acionista com influência significativa nos termos da legislação societária, seja pela presença de membros no conselho de administração com algum tipo de conexão política.

Inicialmente, foi identificado que as conexões políticas por estrutura de propriedade ocorrem em 15 das 26 empresas que compõem a amostra de pesquisa, nas quais foi possível observar médias anuais de capital votante do governo superiores a $60 \%$, o que indica que há um forte controle em algumas empresas do segmento de energia elétrica.

A tabela 5 apresenta as estatísticas descritivas da conectividade política por meio da estrutura de propriedade. 
Tabela 5 - Conexões políticas por estrutura de propriedade

\begin{tabular}{c|c|c|c|c|c|c}
\hline \multirow{2}{*}{$\begin{array}{c}\text { Capital votante do } \\
\text { governo acionista }\end{array}$} & $\mathbf{2 0 1 3}$ & $\mathbf{2 0 1 4}$ & $\mathbf{2 0 1 5}$ & $\mathbf{2 0 1 6}$ & $\mathbf{2 0 1 7}$ & $\mathbf{2 0 1 8}$ \\
\cline { 2 - 7 } & 0,6412 & 0,6389 & 0,6389 & 0,6389 & 0,6465 & 0,6232 \\
Média & 0,6516 & 0,6516 & 0,6516 & 0,6516 & 0,6516 & 0,6190 \\
Mediana & 0,2414 & 0,2426 & 0,2426 & 0,2426 & 0,2376 & 0,2335 \\
Desvio padrão & 0,3765 & 0,3797 & 0,3797 & 0,3797 & 0,3676 & 0,3747 \\
Coeficiente de variação & 0,2396 & 0,2396 & 0,2396 & 0,2396 & 0,2194 & 0,1980 \\
Mínimo & 0,9761 & 0,9761 & 0,9761 & 0,9761 & 0,9761 & 0,9761 \\
\hline
\end{tabular}

Fonte: dados da pesquisa (2021)

Conforme os dados apresentados na tabela 5, o percentual mínimo observado de participação na propriedade das empresas foi de $19,80 \%$ em 2018, ao passo que nos demais períodos esse percentual foi superior a $21,94 \%$. Esse resultado indica que mesmo no mínimo, nos termos da legislação societária, o governo exerce influência significativa sobre as empresas do segmento de energia elétrica.

Na sequência, foram examinadas as composições dos conselhos de administração das companhias componentes da amostra. Na tabela 6, indica-se o percentual de empresas em que foi identificado pelo menos um membro do conselho de administração com algum tipo de conectividade política ao longo do período estudado.

Tabela 6 -Conectividade Política em Conselhos de Administração

\begin{tabular}{c|c|c|c|c|c|c}
\hline \multirow{2}{*}{$\begin{array}{c}\text { Conexão Política pelo } \\
\text { Conselho de Administração }\end{array}$} & \multicolumn{7}{|c}{ Ano } \\
\cline { 2 - 7 } & $\mathbf{2 0 1 3}$ & $\mathbf{2 0 1 4}$ & $\mathbf{2 0 1 5}$ & $\mathbf{2 0 1 6}$ & $\mathbf{2 0 1 7}$ & $\mathbf{2 0 1 8}$ \\
\hline Empresas conectadas & $27,27 \%$ & $27,27 \%$ & $36,36 \%$ & $18,18 \%$ & $45,45 \%$ & $27,27 \%$ \\
Empresas não conectadas & $72,73 \%$ & $72,73 \%$ & $63,64 \%$ & $81,82 \%$ & $54,55 \%$ & $72,73 \%$ \\
Total & $100,00 \%$ & $100,00 \%$ & $100,00 \%$ & $100,00 \%$ & $100,00 \%$ & $100,00 \%$ \\
\hline
\end{tabular}

Fonte: dados da pesquisa (2021)

Destacam-se que os exercícios de 2015 e 2017 (os quais antecederam anos eleitorais para prefeito e presidente e governador, respectivamente) são superiores aos demais. Uma possível explicação estaria em Baysinger (1984), o qual reforça essa prática como uma tentativa da organização em usar os poderes do governo para perseguir interesses particulares dos proprietários a fim de gerenciar possíveis ameaças governamentais e de monitorar possíveis ameaças concorrenciais.

Desse modo, o primeiro objetivo de pesquisa é alcançado ao se identificar que há indícios de conectividade política pelo fato de haver uma forte participação societária do governo na estrutura de propriedade das companhias do segmento de energia elétrica. Ademais, também foi observado o valor mínimo de 18,18\% no ano de 2016 em que as empresas componentes da amostra apresentam em sua composição pelo menos um membro do conselho de administração que é ex-político.

\subsection{Análise de Regressão}

Na presente subseção é realizada a análise de regressão quantílica com a finalidade de descrever as relações entre o desempenho econômico e a conectividade política.

Na tabela 7 são apresentados os resultados da regressão quantílica estimada para os quantis ( $\tau$ ) 0,25 ; 0,50; e 0,75, respectivamente, em função da variável Conexão Geral (ConexG), quando há conexão ou por estrutura de propriedade ou presença em conselhos de administração. 
Tabela 7 -Regressão quantílica com a variável independente ConexG

\begin{tabular}{|c|c|c|c|c|c|c|}
\hline \multirow{2}{*}{ Variáveis } & \multicolumn{2}{|c|}{$\tau(0,25)$} & \multicolumn{2}{|c|}{$\tau(0,50)$} & \multicolumn{2}{|c|}{$\tau(0,75)$} \\
\hline & QTobin & $M T B$ & QTobin & $M T B$ & QTobin & $M T B$ \\
\hline Intercepto & $-4,63895^{\star}$ & $-13,77083^{\star \star}$ & $-9,33039 * \star \star$ & $-29,00290 * \star \star$ & $-15,4411^{\star \star \star}$ & $-43,4313^{\star \star \star *}$ \\
\hline ConexG & $-0,03776$ & $-0,27849$ & 0,29708 & 0,65792 & $0,86209 * \star$ & 1,67738 ** \\
\hline TAM & 0,30084 & $0,97395^{\star \star}$ & $0,65946^{\star \star \star}$ & $1,98715^{\star \star \star}$ & $1,23573^{\star \star \star}$ & 3,19372 \\
\hline ALAV & 0,34897 & $-0,06315$ & $-0,31550$ & 0,88725 & $-3,57852^{\star \star}$ & $-3,08775^{\star \star \star}$ \\
\hline LC & 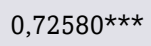 & $0,07765^{\star \star \star}$ & 0,70218 *** & $0,74570 \star \star \star$ & $0,83879 * \star \star$ & $0,08285^{\star \star \star}$ \\
\hline GC & $-0,59829 * \star$ & $-1,32493^{\star \star \star}$ & $-0,86553^{\star \star *}$ & $-2,04198^{\star \star *}$ & $-1,30844^{\star \star \star}$ & $-3,09763^{\star \star *}$ \\
\hline
\end{tabular}

Fonte: dados da pesquisa (2021)

Para essa estimação, a variável Conex $G$ não se mostrou significante para os quantis $\tau=0,25$ e 0,50 . Por outro lado, $\tau$ $>$ 0,75 foi possível observar relações significantes e positivas para com o desempenho econômico mensurado tanto pelo $Q$ de Tobin quanto pelo Market-to-Book.

A observação dos resultados supramencionados permite inferir que a conexão política, quando mensurada pela simples presença do acionista governo ou pela presença de membro de conselho de administração com algum tipo de conectividade política só está relacionada com níveis mais elevados de desempenho econômico, o que converge com os resultados dos trabalhos de Hillman (2005), Camilo et al. (2012) e Jackowicz et al. (2014) indicando que não é possível rejeitar a $H_{1}$ de que há uma relação positiva entre a conectividade política e o desempenho econômico.

Tomando por base os resultados observados e o corpo de evidências empíricas, é possível inferir que a conectividade política pode resultar em um acesso preferencial a recursos e capitais, a benefícios fiscais, ao favorecimento em contratos com o governo, e à flexibilização de regulações (Faccio, 2006), o que leva a um alinhamento de interesses entre as partes da relação contratual de agência. No entanto, a conectividade política pode contribuir para que as companhias do segmento de energia elétrica da B3 tenham uma maior dependência das políticas adotadas pelo governo, situação esta que a depender das intenções futuras do governo (agente) pode resultar em conflitos de agência em relação aos demais acionistas e credores (principal).

No que diz respeito à análise das variáveis de controle, as variáveis tamanho e liquidez corrente se mostraram significantes para todos os quantis analisados, exceto para $\tau=0,25$ da regressão com a variável dependente $Q$ de Tobin e o $\tau=0,75$ da regressão com variável dependente Market-to-Book, apresentando um sinal positivo conforme o esperado, indicando que empresas maiores e com melhor liquidez tenham maior propensão de apresentar um melhor desempenho econômico. A variável alavancagem exibiu um sinal negativo, conforme as predições teóricas (Okimura et. al., 2007; Boubakri et al., 2008), porém, apenas para o $\tau=0,75$, confirmando que maiores níveis de endividamento contribuem para um pior desempenho econômico.

Por outro lado, a variável governança corporativa, divergindo do comportamento esperado, se mostrou negativamente relacionada ao desempenho econômico para todos os quantis analisados. Esse resultado sugere indícios de que melhores práticas de governança corporativa não necessariamente resultam em um maior desempenho econômico para o setor elétrico. Uma possível explicação para a observação desse resultado é o de que o mercado de capitais brasileiro é caracterizado pela concentração de propriedade e pela fraca proteção legal ao investidor minoritário (enforcement) (LA PORTA et al., 1998, 2000), fato este corroborado pelos resultados da presente pesquisa, onde foram constatadas médias superiores a $60 \%$ para o percentual de capital votante do governo durante o período analisado (tabela 5). Desse modo, é possível que a observação de um sinal negativo para a relação supramencionada seja dada em função da percepção de que o mercado não enxerga que melhores práticas de governança corporativa sejam capazes de inibir comportamentos oportunistas da gestão (nomeada pelo governo) e de reduzir o nível de incerteza quanto ao surgimento de conflitos de agência derivados de problemas de assimetria informacional.

Na sequência, conforme os procedimentos metodológicos já relatados, a variável de conexão política é desmembrada em duas. Na tabela 8, são apresentados os resultados da estimação quantílica com a variável de conexão política por presença na estrutura de propriedade (ConexPro). 
Tabela 8 - Regressão quantílica com a variável independente ConexPro

\begin{tabular}{|c|c|c|c|c|c|c|}
\hline \multirow{2}{*}{ Variáveis } & \multicolumn{2}{|c|}{$\tau(0,25)$} & \multicolumn{2}{|c|}{$\tau(0,50)$} & \multicolumn{2}{|c|}{$\tau(0,75)$} \\
\hline & QTobin & MTB & QTobin & MTB & QTobin & MTB \\
\hline Intercepto & $-4,86417^{\star \star \star}$ & $-13,53200^{*}$ & $-9,12593^{\star \star \star}$ & 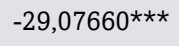 & $-15,06734^{\star \star \star}$ & $-43,5091^{\star * *}$ \\
\hline ConexPro & $0,19089 * \star$ & $-0,15874$ & $0,39218^{*}$ & $1,01755^{\star}$ & 0,80821 ** & $2,24783^{\star \star \star}$ \\
\hline TAM & 0,29931 & $0,96277^{\star \star *}$ & $0,62971^{\star \star \star}$ & 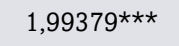 & $1,21718^{\star \star \star}$ & $3,33613^{* * *}$ \\
\hline ALAV & 0,72153 & $-0,17219$ & 0,01733 & 0,86729 & $-3,44772^{\star \star \star}$ & $-6,23533$ \\
\hline LC & $0,07256^{\star \star \star}$ & $0,77294^{\star \star \star}$ & $0,70493^{\star \star \star}$ & $0,73778^{\star \star \star}$ & $0,08373^{\star \star \star}$ & $0,74825^{\star \star \star}$ \\
\hline GC & $-0,66966^{\star \star \star}$ & $-1,44933^{\star \star *}$ & $-0,81362^{\star \star \star}$ & $-2,28771^{\star \star \star}$ & $-1,44828^{\star \star *}$ & $-3,01710 * * *$ \\
\hline
\end{tabular}

Fonte: dados da pesquisa (2021)

A partir dos dados evidenciados na tabela 8 é possível perceber que a variável ConexPro apresentou resultados estatisticamente significantes com sinal positivo e com coeficientes crescentes em todos os quantis analisados, exceto para $\tau=0,25$ da regressão com variável dependente Market-to-Book. Esse resultado indica que a conexão política por estrutura de propriedade resulta em um melhor desempenho econômico, convergindo para a não rejeição da $\mathrm{H}_{1 \mathrm{a}}$ de que há uma relação positiva entre conectividade política por meio do acionista governo com o desempenho econômico das empresas do segmento de energia elétrica listadas na B3.

Indo além, conforme evidenciado na subseção 4.1 da presente pesquisa, há concentração de propriedade por parte do governo, o que permite inferir que a presença do governo enquanto acionista com influência significativa ou como controlador resultou em um maior desempenho econômico das companhias do segmento de energia elétrica. Desse modo, este resultado pode ser explicado pela capacidade do governo de nomear administradores e membros do conselho que votem de acordo com os objetivos e metas institucionais (Hillman \& Hitt, 1999) que levaram a um melhor desempenho econômico por meio de acesso a facilidades oferecidas pelo governo, como, por exemplo, o acesso a fontes externas de financiamento com taxas subsidiadas, reduções de carga tributária por meio de isenções ou até mesmo a celebração de contratos de longo prazo que se transformem em vantagens concorrenciais (Faccio, 2006), resultando, assim, em um alinhamento, na relação contratual de agência, entre agente (governo) e principal (demais acionistas e credores).

No que diz respeito às variáveis de controle, foram observadas relações com sinais similares a estimação do modelo anterior, sendo, portanto, oferecidas as mesmas explicações para os resultados obtidos.

Por conseguinte, foi estimada a regressão quantílica para a variável de conexão política por meio do conselho de administração (ConexPre). Na tabela 9 são apresentados os resultados da referida estimação.

Tabela 9 - Regressão quantílica com a variável independente ConexPre

\begin{tabular}{|c|c|c|c|c|c|c|}
\hline \multirow{2}{*}{ Variáveis } & \multicolumn{2}{|c|}{$\tau(0,25)$} & \multicolumn{2}{|c|}{$\tau(\mathbf{0 , 5 0 )}$} & \multicolumn{2}{|c|}{$\tau(0,75)$} \\
\hline & QTobin & MTB & QTobin & $M T B$ & QTobin & MTB \\
\hline Intercepto & $-4,32030 *$ & $-15,98133^{\star *}$ & $-7,81793^{\star \star \star}$ & $-28,20212^{\star \star \star}$ & 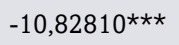 & $-30,6525^{\star \star \star}$ \\
\hline ConexPre & $-0,12699$ & $-0,38644$ & $-0,05015$ & $-0,88865$ & $-0,43466$ & $-1,57662^{\star \star}$ \\
\hline TAM & 0,27004 & 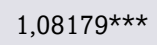 & 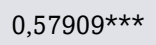 & $2,01190 * \star \star$ & $0,97837 * \star \star$ & 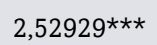 \\
\hline ALAV & 0,53325 & 0,87301 & $-0,43223$ & $-0,47643$ & $-3,93340 * \star$ & $-4,90981$ \\
\hline LC & $0,72797^{\star \star \star}$ & $0,78025^{\star \star \star}$ & $0,70303 * \star \star$ & $0,73722^{\star \star \star}$ & $0,84605^{\star \star \star}$ & 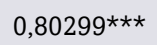 \\
\hline GC & $-0,51876^{\star \star \star}$ & 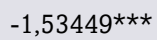 & 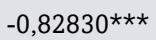 & $-1,82703^{* \star \star}$ & $-1,14596^{\star \star \star}$ & $-3,24622^{\star \star \star}$ \\
\hline
\end{tabular}

Fonte: dados da pesquisa (2021)

É possível observar que a variável ConexPre não apresentou relação estatisticamente significante em relação as variáveis dependentes, exceto para o $\tau=0,75$ da regressão de variável dependente Market-to-Book. Diante desses resultados, não é possível concluir que exista relação entre este tipo de conexão política e o desempenho econômico das companhias do segmento de energia elétrica. Uma possível explicação para este resultado é que foi analisada apenas a presença de membros do conselho de administração que são considerados politicamente conectados. Isto é, é possível que o poder exercido pelo governo sobre os conselhos de administração das companhias do segmento de energia elétrica da B3 seja exercido por meio da presença de conselheiros que não preencheram os requisitos para ser considerado como politicamente conectados ou meramente pelo poder de voto conferido pela concentração de propriedade. 
Sendo assim, é possível rejeitar parcialmente $\mathrm{H}_{1 \mathrm{~b}}$ posto que não foram encontradas evidências robustas de que exista conexão política por meio do conselho de administração, o que diverge das evidências empíricas apresentadas nos estudos de Ecco (2010) e Chen et al. (2011). Como reflexão teórica para este resultado, é possível que a não significância dessa variável (para quase todos os quantis analisados) esteja relacionada à impossibilidade, por parte dos controladores, de obtenção de benefícios pela simples presença no conselho de administração, o que reforça que a conexão política preponderante se dê por meio da concentração acionária (Thomsen \& Pedersen, 2000) e seja exercida pelos direitos de voto.

Por fim, as variáveis de controle apresentaram significância estatística e sinais semelhantes aos das duas estimações realizadas anteriormente, sendo dadas a eles a mesma interpretação.

\section{CONCLUSÃO}

O objetivo deste estudo foi verificar a relação entre a conectividade política e o desempenho econômico das empresas do segmento de energia elétrica listadas na B3. A conectividade política, numa visão geral, mostrou-se positivamente relacionada ao desempenho econômico apenas para $\tau>0,75$, confirmando parcialmente a primeira hipótese do estudo ao demonstrar que há relação entre a conectividade política e o desempenho econômico das companhias do segmento de energia elétrica da B3.

No desdobramento da conexão política por estrutura de propriedade e presença em conselhos de administração, os resultados evidenciaram que a conexão por propriedade se mostrou significante e positivamente relacionada para todos os quantis, exceto para o $\tau=0,25$ quando o desempenho econômico foi mensurado pela variável Market-to-Book. Ademais, a conexão por propriedade apresentou um comportamento crescente do seu coeficiente ao longo dos quantis, sugerindo indícios de que quanto mais intensa for a conectividade política, maior a propensão das companhias do seguimento de energia elétrica da B3 alcançar um maior desempenho econômico.

Por outro lado, a variável conexão por presença no conselho de administração apresentou resultados significativos apenas para o $\tau=0,75$ quando o desempenho econômico é mensurado pela variável Market-to-Book, permitindo rejeitar a hipótese de que a presença de indivíduos com alguma conexão política nos conselhos de administração possa estar relacionada com um melhor desempenho econômico.

Diante dos achados observados, a presente pesquisa contribui com evidências empíricas de que o desempenho econômico das companhias do segmento de energia elétrica da B3 é positivamente relacionado à participação do governo como acionista. Com este achado, vislumbra-se a oportunidade de instigar o debate sobre a concentração acionária do governo nos segmentos de serviços e utilidade pública, sobretudo, com a finalidade de compreender de forma mais abrangente como a participação estatal interfere (seus efeitos) nas decisões estratégicas e, por consequência, na continuidade operacional das entidades dos referidos segmentos no que diz respeito à relação contratual de agência entre o governo e os demais acionistas.

Desse modo, para pesquisas futuras, é possível sugerir que a participação estatal seja estudada no contexto de governança corporativa das companhias do segmento de energia elétrica e dos demais segmentos de serviços e utilidade pública da B3, de forma a possibilitar a análise dos efeitos da participação estatal na estrutura de propriedade e, com isso, esclarecer como ocorre na prática a relação observada neste estudo.

\section{REFERÊNCIAS}

Arrow, K. J. (1985). Principals and agents: the structure of business. Harvard business school press.

Bandeira-de-Mello, R., \& Marcon, R. (2011). The value of business group affiliation for political connections: preferential lending in Brazil. XXèmeConférence de l'AIMS (Association Internationale de ManagmentStrategique).

Barringer, B. R., \& Harrison, J. S. (2000). Walking a tightrope: Creating value through interorganizational relationships. Journal of management, 26(3), 367-403.https://doi.org/10.1177/014920630002600302

Baysinger, B. D. (1984). Domain maintenance as an objective of business political activity: An expanded typology. Academy of Management Review, 9(2), 248-258.

Berle, A., \& Means, G. (1932). The Modern Corporation and Private Property Macmillan. New York, 2(3), 45-53.

Brasil. (2016). 6.404, de 15 de dezembro de 1976. Dispõe sobre as Sociedades por Ações. Disponivel em: Acesso em, 10(09).. Disponivel: <http://www.planalto.gov.br/ccivil_03/leis/16404consol.htm>. Acesso em 02 abr. 2019

Brasil. (1997) 9.504, DE 30 DE SETEMBRO DE 1997.Estabelece normas para as eleições. Disponível: <http://www.planalto.gov. br/ccivil_03/leis/19504.htm>. Acesso em 27 abr. 2019

Brey, N. K., Camilo, S. P. O., Marcon, R., \& Alberton, A. (2011). A estrutura de propriedade das corporações: conexões políticas sob a perspectiva da dependência de recursos. Revista Ibero Americana de Estratégia, 10(3), 126-146. DOI 10.5585/riae.v10i3.1791

Brey, N. K., Camilo, S. P. O., Marcon, R., \& Bandeira-de-Mello, R. (2014). Conexões políticas em estruturas de propriedade: o governo como acionista em uma análise descritiva.. http://dx.doi.org/10.1590/S1678-69712014000100005.

Boubakri, N., Cosset, J. C., \& Saffar, W. (2008). Political connections of newly privatized firms. Journal of corporate finance, 14(5), 654-673. https://doi.org/10.1016/j.jcorpfin.2008.08.003 
Camilo, S. P. O., Marcon, R., \& Bandeira-de-Mello, R. (2012). Conexões políticas e desempenho: um estudo das firmas listadas na BM\&FBovespa. Rev. adm. contemp, 806-826. doi:https://doi.org/10.1590/S1415-65552012000600003.

Caixe, D. F., \& Krauter, E. (2013). A influência da estrutura de propriedade e controle sobre o valor de mercado corporativo no Brasil. Revista Contabilidade \& Finanças, 24(62), 142-153. http://dx.doi.org/10.1590/S1519-70772013000200005.

Chen, C. J., Li, Z., Su, X., \& Sun, Z. (2011). Rent-seeking incentives, corporate political connections, and the control structure of private firms: Chinese evidence. Journal of Corporate Finance, 17(2), 229-243. https://doi.org/10.1016/j.jcorpfin.2010.09.009

Chen, H., Parsley, D., \& Yang, Y. W. (2015). Corporate lobbying and firm performance. Journal of Business Finance \& Accounting, 42(3-4), 444-481. https://doi.org/10.1111/jbfa.12109

Chung, K. H., \& Pruitt, S. W. (1994). A simple approximation of Tobin's q. Financial management, 70-74. doi:10.2307/3665623

Claessens, S., Feijen, E., \& Laeven, L. (2008). Political connections and preferential access to finance: The role of campaign contributions. Journal of financial economics, 88(3), 554-580. https://doi.org/10.1016/j.jfineco.2006.11.003

Cooper, M. J., Gulen, H., \& Ovtchinnikov, A. V. (2010). Corporate political contributions and stock returns. The Journal of Finance, 65(2), 687-724.

Correia, T. D. S. (2018). Governança corporativa, princípios globais de contabilidade gerencial e código de ética empresarial de empresas brasileiras de capital aberto.

Dinç, I. S. (2005). Politicians and banks: Political influences on government-owned banks in emerging markets. Journal of financial economics, 77(2), 453-479. https://doi.org/10.1016/j.jfineco.2004.06.011

Ecco, I. L. (2010). Conselhos de administração das empresas reguladas no brasil: conexoes politicas e o seu desempenho. Disponível em: <https://siaiap39.univali.br/repositorio/handle/repositorio/1645>

Faccio, M. (2006). Politically connected firms. American economic review, 96(1), 369-386.DOI: 10.1257/000282806776157704

Faccio, M., \& Parsley, D. C. (2009). Sudden deaths: Taking stock of geographic ties. Journal of Financial and Quantitative Analysis, 44(3), 683-718. doi:10.2139/ssrn.875808

Fich, E. M., \& White, L. J. (2005). Why do CEOs reciprocally sit on each other's boards?. Journal of Corporate Finance, 11(1-2), 175-195.https://doi.org/10.1016/j.jcorpfin.2003.06.002

Hillman, A. J. (2005). Politicians on the board of directors: Do connections affect the bottom line?. Journal of management, 31(3), 464-481.https://doi.org/10.1177/0149206304272187

Hillman, A. J., \& Hitt, M. A. (1999). Corporate political strategy formulation: A model of approach, participation, and strategy decisions. Academy of management review, 24(4), 825-842. doi:10.2307/259357

Hillman, A. J., Zardkoohi, A., \& Bierman, L. (1999). Corporate political strategies and firm performance: indications of firm-specific benefits from personal service in the US government. Strategic Management Journal, 20(1), 67-81. https://doi.org/10.1002/(SICI)1097-0266(199901)20:1<67::AID-SMJ22>3.0.CO;2-T

Hill, M. D., Fuller, K. P., Kelly, G. W., \& Washam, J. O. (2014). Corporate cash holdings and political connections. Review of Quantitative Finance and Accounting, 42(1), 123-142.

Jackowicz, K., Kozłowski, Ł., \& Mielcarz, P. (2014). Political connections and operational performance of non-financial firms: New evidence from Poland. Emerging Markets Review, 20, 109-135. doi:10.1016/j.ememar.2014.06.005

Jensen, M. C., \& Meckling, W. H. (1979). Theory of the firm: Managerial behavior, agency costs, and ownership structure. In Economics social institutions (pp. 163-231). Springer, Dordrecht. https://doi.org/10.1016/0304-405X(76)90026-X

Kerr, G., \& Darroch, J. (2004). Performance in the contemporary conglomerate. Academy of Strategic Management Journal, 3, 21.

Koenker, R., \& Bassett Jr, G. (1978). Regression quantiles. Econometrica: journal of the Econometric Society, 33-50.doi: $10.2307 / 1913643$

Koprowski, S., Krein, V., Barichello, R., Mazzioni, S., \& Dal Magro, C. B. (2019). Influência das conexões políticas e da evidenciação socioambiental no custo de capital. https://doi.org/10.19094/contextus.v17i2.40358

La Porta, R., Lopez-de-Silanes, F., Shleifer, A., \& Vishny, R. W. (1997). Legal determinants of external finance. The journal of finance, 52(3), 1131-1150.https://doi.org/10.1111/j.1540-6261.1997.tb02727.x

La Porta, R. L., Lopez-de-Silanes, F., Shleifer, A., \& Vishny, R. W. (1998). Law and finance. Journal of political economy, 106(6), 1113-1155.

La Porta, R., Lopez-de-Silanes, F., Shleifer, A., \& Vishny, R. (2000). Investor protection and corporate governance. Journal of financial economics, 58(1-2), 3-27..https://doi.org/10.1016/S0304-405X(00)00065-9

La Porta, R., Lopez-de-Silanes, F., \& Shleifer, A. (2002). Government ownership of banks. The Journal of Finance, 57(1), $265-301$. (DOI): 10.3386/w7620

Macêdo, J. M. A., Silva, C. A. T., \& Machado, M. A. V. (2015). Conexões políticas e as empresas brasileiras: um estudo experimental sobre as decisões de investimento no mercado de capitais. Advances in Scientific and Applied Accounting, 8(2), 157-178. http://dx.doi. org/10.14392/asaa.2015080202

Macêdo, J. M. A. (2017). Conexões políticas e desempenho: um estudo da mutualidade entre políticos, governo e bancos em operação no Brasil. 
Menozzi, A., Gutiérrez Urtiaga, M., \& Vannoni, D. (2011). Board composition, political connections, and performance in state-owned enterprises. Industrial and Corporate Change, 21(3), 671-698..https://doi.org/10.1093/icc/dtr055

Okimura, R. T., Silveira, A. D. M. D., \& Rocha, K. C. (2007). Estrutura de propriedade e desempenho corporativo no Brasil. RAC-Eletrônica, 1(1), 119-135.

Pinheiro, B. G., De Luca, M. M. M., \& Vasconcelos, A. C. D. (2016). Conexões políticas nas maiores companhias listadas na BM\&FBovespa. REAd. Revista Eletrônica de Administração (Porto Alegre), 22(2), 394-418. http://dx.doi.org/10.1590/1413-2311.1622015.60360.

Salamon, L. M., \& Siegfried, J. J. (1977). Economic power and political influence: The impact of industry structure on public policy. American Political Science Review, 71(3), 1026-1043. DOI: 10.2307/1960105

Shaffer, B. (1995). Firm-level responses to government regulation: Theoretical and research approaches. Journal of management, 21(3), 495-514. doi:10.1177/014920639502100305

Shleifer, A., \& Vishny, R. W. (1997). A survey of corporate governance. The journal of finance, 52(2), 737-783. https://doi.org/10.1111/j.1540-6261.1997.tb04820.x

Silva, J. C. D., Xavier, W. G., Gambirage, C., \& Camilo, S. P. O. (2018). A Influência das Conexões Políticas no Custo de Capital e no Desempenho das Empresas Listadas na B3. BBR. Brazilian Business Review, 15(4), 317-330. https://doi.org/10.15728/bbr.2018.15.4.1

Thomsen, S., \& Pedersen, T. (2000). Ownership structure and economic performance in the largest European companies. Strategic Management Journal, 21(6), 689-705. https://doi.org/10.1002/(SICI)1097-0266(200006)21:6\%3C689::AID-SMJ115\%3E3.0.CO;2-Y

Wu, W., Wu, C., \& Rui, O. M. (2012). Ownership and the value of political connections: Evidence from China. European Financial Management, 18(4), 695-729. doi:10.1111/j.1468-036x.2010.00547.x 